\title{
Alice in Wonderland Syndrome- Like Seizure and Refractory Supraventricular Tachycardia
}

\author{
Pitirat Panpruang $^{a} \quad$ Monton Wongwandee ${ }^{b} \quad$ Nattapun Rattanajaruskul $^{c}$ \\ Worawut Roongsangmanoon ${ }^{\mathrm{C}}$ Arthit Wongsoasu ${ }^{\mathrm{C}}$ \\ Teeranan Angkananard ${ }^{c}$ \\ aDepartment of Medicine, HRH Princess Maha Chakri Sirindhorn Medical Center, Faculty \\ of Medicine, Srinakharinwirot University, Ongkharak, Nakhon Nayok, Thailand; ${ }^{b}$ Division of \\ Neurology, Department of Medicine, HRH Princess Maha Chakri Sirindhorn Medical Center, \\ Faculty of Medicine, Srinakharinwirot University, Ongkharak, Nakhon Nayok, Thailand; \\ 'Division of Cardiovascular Medicine, Department of Medicine, HRH Princess Maha Chakri \\ Sirindhorn Medical Center, Faculty of Medicine, Srinakharinwirot University, Ongkharak, \\ Nakhon Nayok, Thailand
}

\section{Keywords}

Alice in Wonderland $\cdot$ Macropsia · Seizure $\cdot$ Tachycardia

\begin{abstract}
Alice in Wonderland syndrome (AIWS) is a rarely curious visual perceptual disorder which has been associated with diverse neurologic and psychiatric problems. It may be a manifestation in migraine, epileptic seizures, encephalitis, other brain lesions, medication-related side effects, schizophrenia, and depressive disorders. Principal character of AIWS is the disproportion between the external world and the self-image in which micropsia (objects appear smaller), macropsia (objects appear larger), and teleopsia (objects appear further away) are frequently reported. The cases of temporal lobe epilepsy may present with complex visual auras of visual distortions (e.g., micropsia and macropsia) like AIWS. We report an unusual case of an elderly man who presented with AIWS, focal impaired awareness seizures, ictal tachyarrhythmia, multiple episodes of transient visual disturbances of macropsia and transient loss of consciousness. During those symptoms, telemetry showed self-limited supraventricular tachycardia several times which could not be regulated with heart rate-controlled medication. The electroencephalography was later tested and showed rhythmic theta activity over the right cerebral hemisphere. He was treated with levetiracetam, and all his symptoms and tachyarrhythmias
\end{abstract}


Panpruang et al:: Alice in Wonderland Syndrome and Tachyarrhythmia

were gradually resolved thereafter. Refractory response to treatment would remind the physicians to reassess for the correct diagnosis.

\section{Introduction}

Alice in Wonderland syndrome (AIWS) [1] is appertaining to visual perception and spatial distortions, principally involving visual and somesthetic integration systems. It was first described by John Todd in 1955. It is a rare visual perceptual disorder which has been associated with diverse neurologic and psychiatric problems. The causes of AIWS are still not clearly known; however, the previous neuroimaging studies showed disturbances of some parts of the brain, involving the temporoparietal junction, the temporal and occipital lobe. It may be a manifestation in migraine, epileptic seizures, encephalitis, other brain lesions, medication-related side effects, schizophrenia, and depressive disorders. Principal character of AIWS is the disproportion between the external world and the self-image in which micropsia (objects appear smaller), macropsia (objects appear larger), and teleopsia (objects appear further away) are frequently reported. This syndrome has no established criteria for diagnosis and can be found at any age but mostly in children. Encephalitides affect mostly in patients younger than 18 years old while the neurologic disorders (migraine, cerebrovascular disorder, and epilepsy) affect mostly in adults and elderly patients.

Macropsia, one type of visual illusion, is an infrequent symptom in which objects are visually perceived to be larger than they are really sized and can be primarily found in retinal pathologies and visual pathway disorders. It would be a manifestation of AWIS types B and C [1], an epileptic seizure, migraine, stroke, and hallucinogenic drugs which usually involve the temporo-parieto-occipital regions [2]. Focal impaired awareness seizures (FIAS), formerly called complex partial seizures with transient loss of consciousness (TLOC), tend to have an aura before the seizures occur. The cases of temporal lobe epilepsy [3] may present with complex visual auras of visual distortions (e.g., micropsia and macropsia) like AIWS.

The clinical manifestations of TLOC reported from a patient and/or a witness may be indistinguishable between seizure and cardiac syncope although their underlying pathophysiology and treatment are quite distinctive. The cardiac syncope may be a consequence of arrhythmia (bradyarrhythmia and tachyarrhythmia) and/or cardiac structural abnormalities. An accurate diagnosis of the underlying etiology is vital for further management and prevention. We report an unusual case of an elderly man who presented with AIWS, FIAS, and ictal tachyarrhythmia which was refractory for heart rate (HR) control and misdiagnosed as cardiac syncope from supraventricular tachycardia (SVT).

\section{Case Report/Case Presentation}

A69-year-old Thai man without an underlying condition presented with multiple episodes of transient visual disturbances of macropsia (seeing things larger than they are) including enhanced stereoscopic vision (an exaggeration of the depth and detail of visually perceived objects). The visual symptoms lasted for a few seconds and were accompanied by impairment of awareness, which his daughter described as not responding to others, for almost a minute. He was seen chewing even though there was no food in his mouth. He has faced those events 2 or 3 times per day for a couple of months and more often in a week before he came to our hospital. He sometimes complained of palpitations as "rapid heartbeat" shortly during 


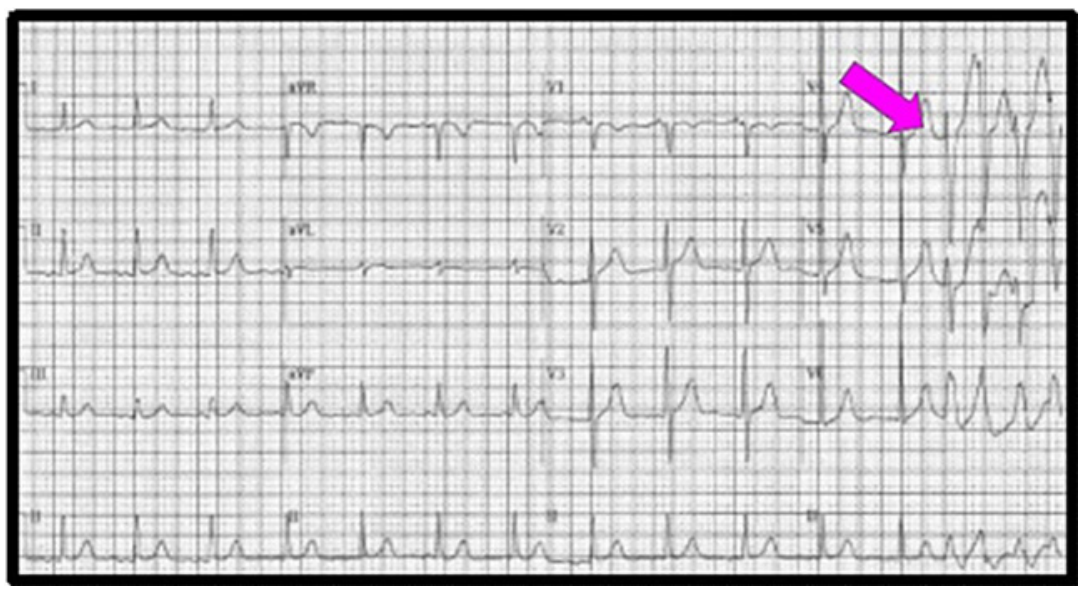

Fig. 1. An initial 12-lead ECG recorded on admission. ECG, electrocardiogram.

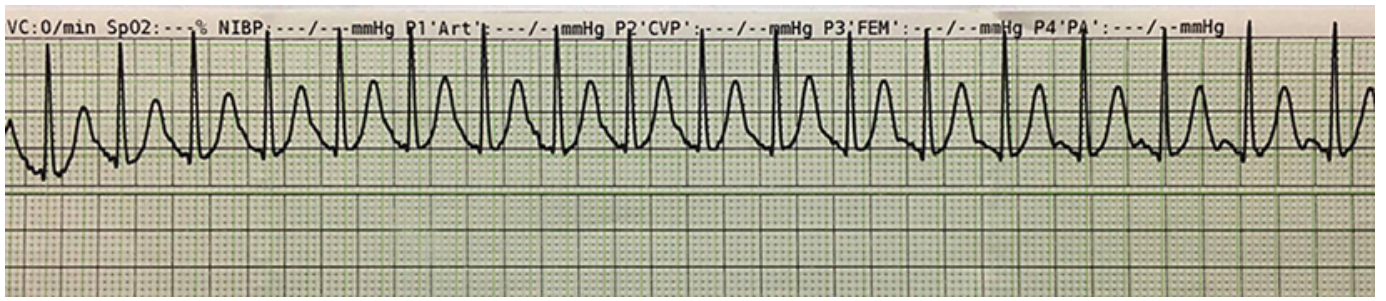

Fig. 2. An ECG strip of narrow complex tachycardia. ECG, electrocardiogram.

the event. He did not recall any dizziness, chest pain, shortness of breath, fever, headache, numbness, or weakness prior to each event. He had never had symptoms like these before. He reported a 35 pack-year history and social drinking. He denied over-the-counter medication abuse, food and drug allergy, and a family history of sudden cardiac death or epilepsy.

On physical examination, the patient revealed afebrile, no tachypnea, good consciousness, and orientation. Initially, his pulse rate was 68 beats $/ \mathrm{min}$ and regular while his blood pressure was $120 / 70 \mathrm{~mm} \mathrm{Hg}$ without orthostatic. The heart sounds were normal without murmurs, and the lungs were clear to auscultation. Significant neurological deficits were not observed. The rest of a thorough multisystem examination was unremarkable.

A 12-lead electrocardiogram (ECG) recorded after the event showed sinus rhythm with a heart rate of 90 beats per minute with a group of wide complex beats (Fig. 1) with suspected nonsustained ventricular tachycardia (VT) [arrow]. Normal QT interval and no significant ST-T change were noted. Therefore, the patient was admitted in an intermediate cardiac care unit for ECG monitoring.

During hospitalization, multiple times of TLOC concomitant of the symptom of AIWS were observed. Telemetry over the next $24 \mathrm{~h}$ showed self-limited multiple episodes of narrow complex tachycardia (Fig. 2) with gradual acceleration followed by deceleration (warm-up and cooldown phenomenon) in which his maximum HR was 161 beats per minute. The suggestive mechanism of that tachycardia was enhanced cardiac automaticity of sinus tachycardia (Fig. 3) or automatic focal atrial tachycardia (AT) (Fig. 4). The P waves which looked similar to those in the normal sinus rhythm indicate sinus tachycardia or focal AT arising close to the sinus node $[4,5]$.

Laboratory study carried out showed a mild microcytic anemia (hemoglobin $9.9 \mathrm{~g} / \mathrm{dL}$ and MCV 54.7 fL). Serum potassium level was $3.5 \mathrm{mmol} / \mathrm{L}$ (reference range: 3.5-5.1 mmol/L), 


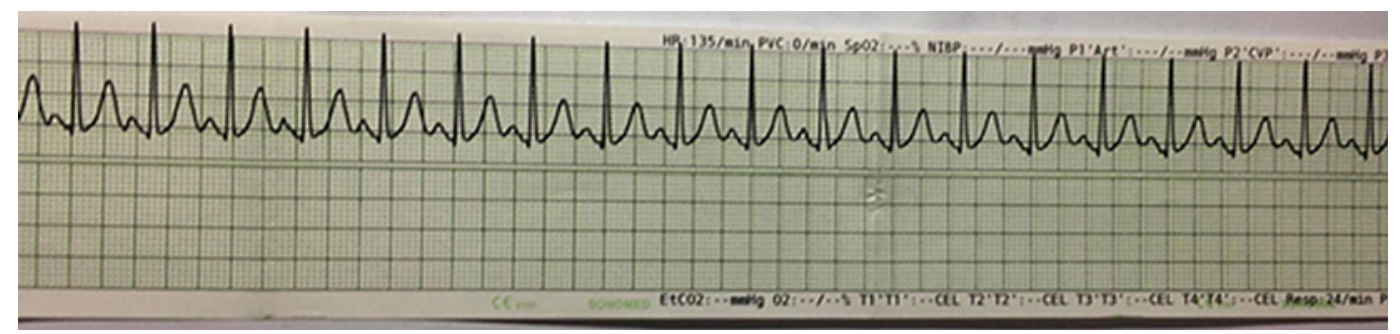

Fig. 3. An ECG strip showing enhanced cardiac automaticity of sinus tachycardia. ECG, electrocardiogram.

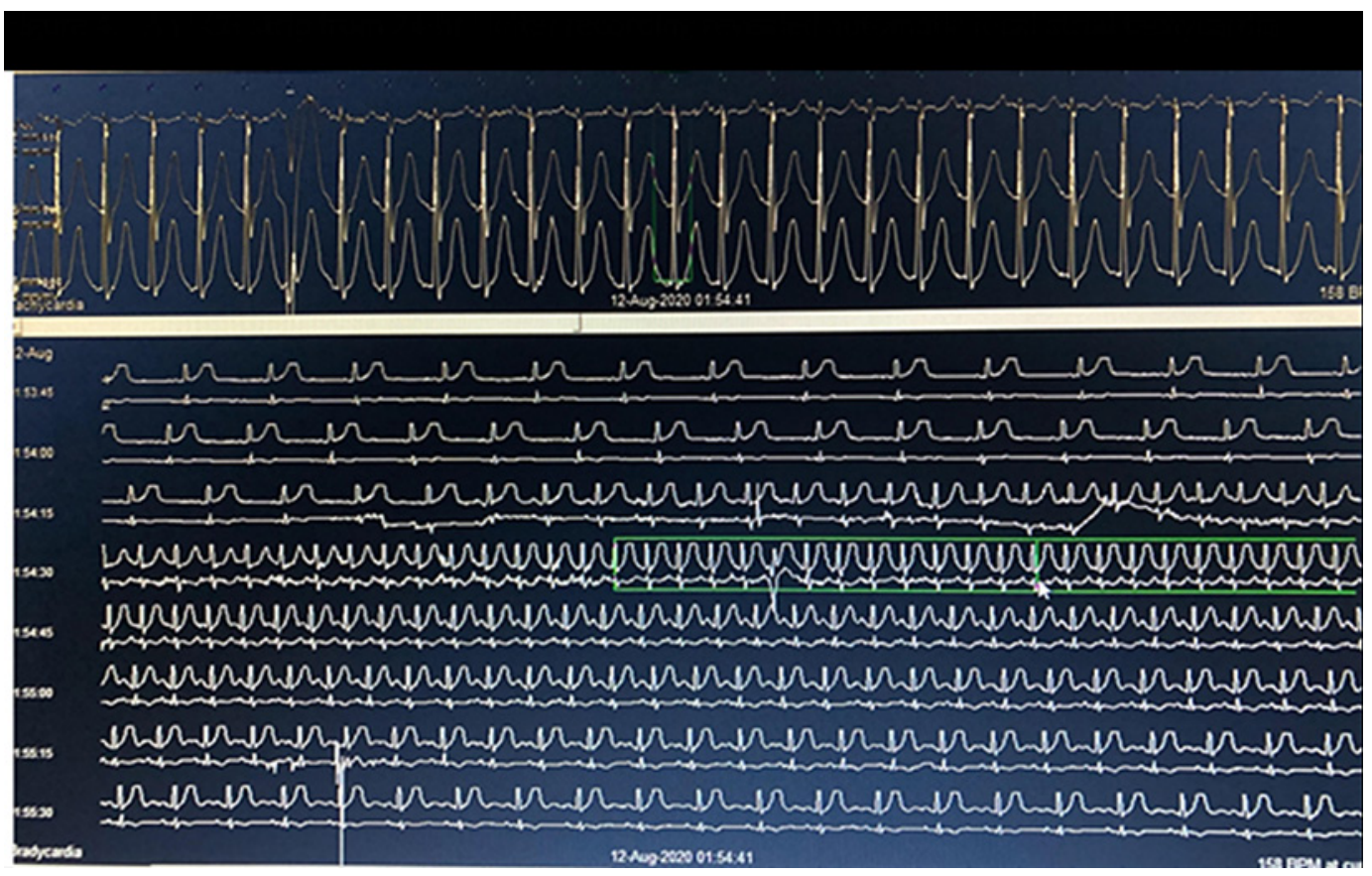

Fig. 4. An ECG strip from 24-h Holter recording revealing automatic focal atrial tachycardia. ECG, electrocardiogram.

calcium $8.9 \mathrm{mg} / \mathrm{dL}$ (reference range: $8.4-10.2 \mathrm{mg} / \mathrm{dL}$ ), magnesium $2.6 \mathrm{mg} / \mathrm{dL}$ (reference range: $1.6-2.6 \mathrm{mg} / \mathrm{dL}$ ), glucose $98 \mathrm{mg} / \mathrm{dL}$, creatinine $0.94 \mathrm{mg} / \mathrm{dL}$ with eGFR by CKD-EPI 82.4 $\mathrm{mL} / \mathrm{min}$ per $1.73 \mathrm{~m}^{2}$, TSH $0.819 \mu \mathrm{IU} / \mathrm{mL}$ (reference range: $0.27-4.2 \mu \mathrm{IU} / \mathrm{mL}$ ), FT3 $2.74 \mathrm{pg} / \mathrm{mL}$ (reference range: $2.00-4.40 \mathrm{pg} / \mathrm{mL}$ ), and FT4 $1.29 \mathrm{ng} / \mathrm{dL}$ (reference range: 0.93-1.70 ng/dL). A transthoracic echocardiogram demonstrated good LV systolic function, normal wall motion, no significant valvular dysfunction, and no pulmonary hypertension.

Five milligrams of bisoprolol was given to control those arrhythmias. However, the patient's symptoms and tachyarrhythmia were frequently noted. Thus, the 30-min scalp electroencephalography (EEG) was done. The patient had one ictal episode showing sudden impairment of consciousness lasting for a minute. He also had lip-smacking and fumbling during the attack. Later, he was able to recall the few-second preictal visual disturbance. The ictal EEG showed rhythmic theta activity (4-5 Hz) over the right cerebral hemisphere for $40 \mathrm{~s}$, accompanied by glossokinetic artifacts (Fig. 5). The entire EEG tracings during his symptom can be followed via the link: https:// drive.google.com/file/d/1yRn4P3YwAgcPYI35J8uu3oP8iOcm6_ku/view?usp=sharing. Sinus tachycardia was also detected during the ictal periods. MRI of the brain revealed multiple discrete patchy T2W FLAIR hyperintense lesions involving bilateral subcortical white matter, which represented small vessel disease. No evidence of acute cerebral infarction, intracerebral hemorrhage, 


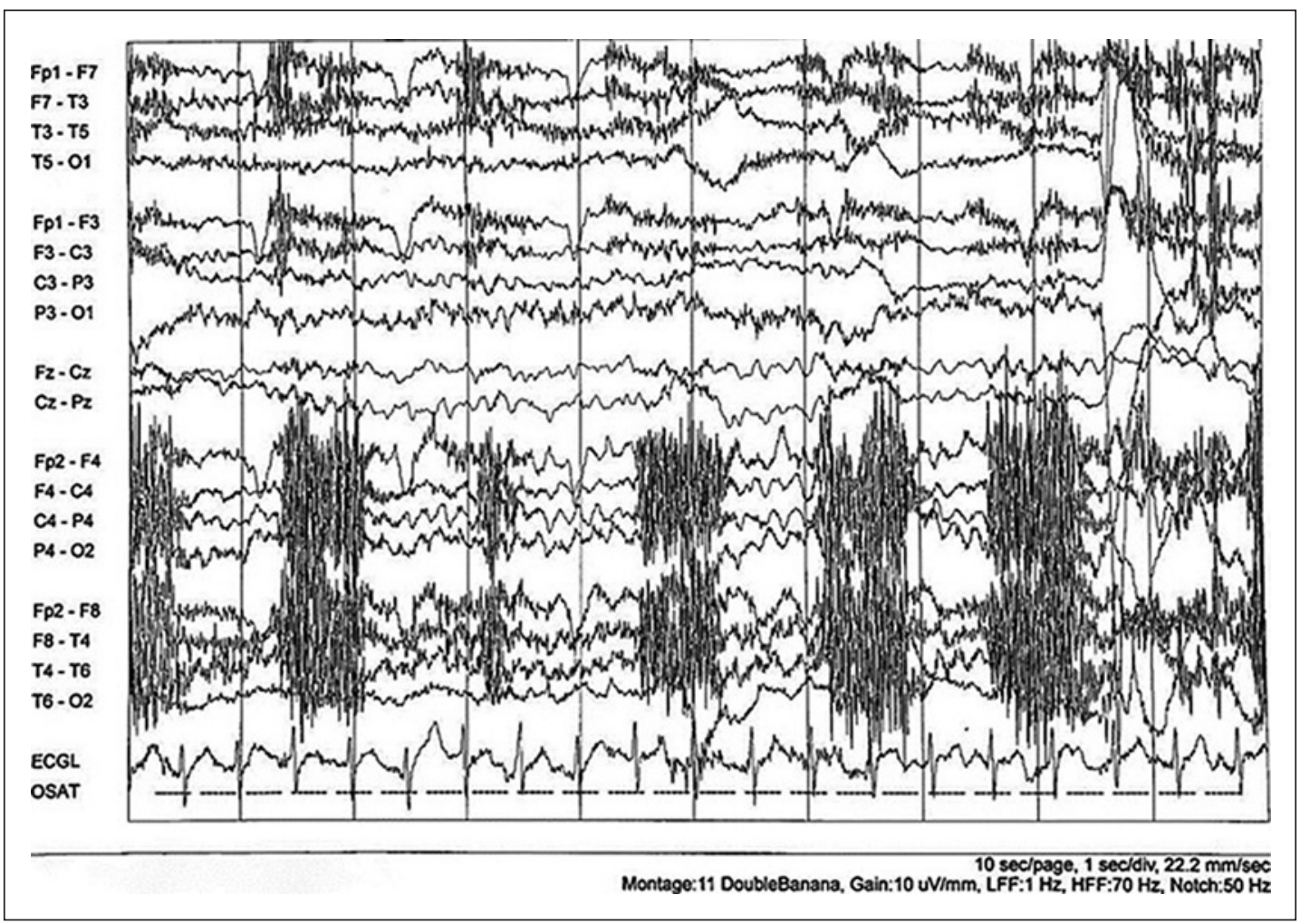

Fig. 5. The scalp EEG showed rhythmical theta (4-5 Hz) activity over the right cerebral hemisphere during the seizure. Electromyography artifacts from oral automatism and sinus tachycardia were recorded. EEG, electroencephalography.

cerebral microbleeds, encephalomalacia, hippocampal atrophy, brain tumor, or vascular malformation was observed. A lumbar puncture was done, and the cerebrospinal fluid profile was normal. Anti-thyroglobulin and anti-microsomal (anti-TPO) antibodies were within normal limits. Serum paraneoplastic antibodies including ANNA-1, ANNA-2, PCA-1, PCA-2, Anti-GFAP, Anti-synaptophysin, AGNA/Anti-SOX1, Anti-Ma (Ma1 and Ma2/Ta), and Anti-amphiphysin were all negative.

Hence, the diagnosis of this patient was focal impaired awareness cognitive and autonomic seizure of unknown etiology. He experienced recurrent episodes of stereotypically TLOC with a preictal phase of macropsia concurrent to episodes of tachyarrhythmias. The differential diagnosis included convulsive syncope induced by tachyarrhythmia. However, all recorded tachyarrhythmia (nonsustained VT, AT, and sinus tachycardia) would not be the cause of the patient's neurological symptoms in which abnormal EEG occurred simultaneously.

He was treated with an antiepileptic drug $\left(2,000 \mathrm{mg}\right.$ per day of levetiracetam [Keppra $\left.\left.{ }^{\circledR}\right]\right)$ and $5 \mathrm{mg}$ of bisoprolol. All the symptoms of macropsia, enhanced stereoscopic vision, TLOC, and tachyarrhythmias were gradually resolved in a period of 4 weeks. There was no documented tachyarrhythmia after discontinuing bisoprolol.

\section{Discussion/Conclusion}

Syncopal TLOC would be due to cerebral global hypoperfusion and caused by cardiac arrhythmias, structural cardiac disorders, orthostatic hypotension, neutrally mediated (reflex) syncope, and FIAS which were formerly known as complex partial seizures. 
Duration of cardiovascular syncope and FIAS would be similar, that is, it may last from a few seconds to a few minutes in cardiac syncope, while the median period of FIAS is 78 (8-298) s [6]. Both syncope and seizures can present with visual disturbances. Visual seizures often manifest with hallucinations varying from unformed images, for example, flashing, to very complex ones [7, 8]. Visual illusions also can occur, including distortions of size, shape, and color. Ictal blindness or visual field defect is also described. However, the visual phenomenon from presyncope is usually described as dimming of light and color, and loss of peripheral vision [9]. Thus, the diagnosis ideally made by both cardiologists and neurologists is dependent on thorough history taking, physical examination, investigations, and closed monitoring.

We report a rare case of FIAS who presented with AIWS-like and ictal tachyarrhythmia which was misdiagnosed as SVT causing syncope. This may be caused by an unusual presentation of FIAS with the aura symptom of visual illusion and thus misled the correct diagnosis. A child with a pure autonomic seizure manifested and misdiagnosed as SVT without improvement by HR-controlled medication [10] has been previously reported. The similarities with prior evidence are an effect of seizure lateralization on increasing HR influence by the right anatomic structures [11] and the patients with FIAS highly associated with an increased HR during the ictal events [12]. The difference between previous case reports presenting with ictal tachycardia (IT) and our case is that IT is likely to be associated with drug-resistant epilepsy [13], whereas it is the initial presentation of ours. The limitation of our report is that the electrophysiologic study for definite diagnosis and type of SVT could not be performed during episodes of the patient's symptoms.

The most common cardiac alterations associated with seizures are the IT with mostly sinus tachycardia and the ictal bradycardia (IB). Various studies of HR variability demonstrate that interictal autonomic alterations are associated with epilepsy. These include combined low parasympathetic tone associated with high sympathetic tone $[14,15]$, inhibition of either the sympathetic or parasympathetic tone [16-18], or inhibition of both sympathetic and parasympathetic tones [19]. Additionally, sinus tachycardia is a normal physiologic response to many conditions in which catecholamine release is physiologically enhanced or, less commonly, in situations where the parasympathetic nervous system is withdrawn. Previous studies have found that during electrical stimulation of the human insular cortex, the right hemisphere may have greater sympathetic influence, while the left hemisphere may be associated with greater parasympathetic control $[17,18]$. The autonomic alterations in epilepsy may be more obvious in patients with right-sided than leftsided epileptic foci $[19,20]$. The IT more frequently occurs with a prevalence of $80-100 \%$ [21], while the IB only affects $<5 \%[22,23]$. Both IT and IB are more frequently found in temporal lobe origin seizure. As to the IT arise, two temporal patterns of ECG have been explored, one with a steady HR increase usually 120-150 bpm and one in which HR increases abruptly and then in a steady continuous manner. IT can occur before or during the seizure period $[11,24]$. There was only 1 reported case with generalized tonic-clonic seizure found SVT in the postictal phase [25]. Several cortical structures with epileptic discharge may connect with autonomic centers from the medulla oblongata, amygdala nucleus, thalamus, and hypothalamus [26] and cause autonomic alterations, as indicated by the peak in catecholamines and electrodermal activity [27].

The present case report demonstrates the importance of careful history taking, clinical examination, and investigation in a case with TLOC which barely differentiates between an epileptic seizure and cardiac syncope. An uncommon presentation of visual illusion and an initial abnormal ECG finding may mislead the right diagnosis. Refractory response to treatment would remind the physicians to reassess for the correct diagnosis. The patient is sometimes required prolonged monitoring or hospitalization and further investigation.

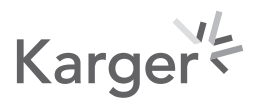


Panpruang et al.: Alice in Wonderland Syndrome and Tachyarrhythmia

\section{Statement of Ethics}

The patient has given his written informed consent to publish his clinical data, electrocardiogram, and electroencephalography. The study protocol conformed to the World Medical Association Declaration of Helsinki and is exempt from ethics committee approval as per the guideline of Srinakharinwirot University given that it is a case report, and informed consent was already obtained.

\section{Conflict of Interest Statement}

The authors have no conflicts of interest to declare.

\section{Funding Sources}

This research received no specific grant from any funding agency in the public, commercial, or not-for-profit sectors.

\section{Author Contributions}

Pitirat Panpruang, Monton Wongwandee, and Teeranan Angkananard co-wrote and edited the manuscript. Nattapun Rattanajaruskul, Worawut Roongsangmanoon, Arthit Wongsoasu, and Teeranan Angkananard contributed to literature review for updating the relevant data.

\section{Data Availability Statement}

All data generated during this study are included in this article.

\section{References}

1 Mastria G, Mancini V, Viganò A, Di Piero V. Alice in wonderland syndrome: a clinical and pathophysiological review. Biomed Res Int. 2016;2016:8243145.

2 Norton JW, Corbett JJ. Visual perceptual abnormalities: hallucinations and illusions. Semin Neurol. 2000; 20(1):111-21.

3 Téllez-Zenteno JF, Hernández-Ronquillo L. A review of the epidemiology of temporal lobe epilepsy. Epilepsy Res Treat. 2012;2012:630853.

4 Brugada J, Katritsis DG, Arbelo E, Arribas F, Bax JJ, Blomström-Lundqvist C, et al. 2019 ESC guidelines for the management of patients with supraventricular tachycardia the task force for the management of patients with supraventricular tachycardia of the European Society of Cardiology (ESC). Eur Heart J. 2020;41(5):655-720.

5 Buttà C, Tuttolomondo A, Di Raimondo D, Milio G, Miceli S, Attanzio MT, et al. Supraventricular tachycardias: proposal of a diagnostic algorithm for the narrow complex tachycardias. J Cardiol. 2013;61(4):247-55.

6 Jenssen S, Gracely EJ, Sperling MR. How long do most seizures last? A systematic comparison of seizures recorded in the epilepsy monitoring unit. Epilepsia. 2006;47(9):1499-503.

7 Manford M, Andermann F. Complex visual hallucinations. Clinical and neurobiological insights. Brain. 1998; 121(Pt 10):1819-40.

8 Panayiotopoulos CP. Elementary visual hallucinations, blindness, and headache in idiopathic occipital epilepsy: differentiation from migraine. J Neurol Neurosurg Psychiatry. 1999;66(4):536-40.

9 Shen WK, Sheldon RS, Benditt DG, Cohen MI, Forman DE, Goldberger ZD, et al. 2017 ACC/AHA/HRS Guideline for the Evaluation and Management of Patients With Syncope: A Report of the American College of Cardiology/ American Heart Association Task Force on Clinical Practice Guidelines and the Heart Rhythm Society. J Am Coll Cardiol. 2017 Aug 1;70(5):e39-e110. 
10 Kim YD, Yang JH, Han SD, Shin HW, Park S. A case of pure autonomic seizure presenting as sinus tachycardia. J Korean Neurol Assoc. 2004;22(6):652-6.

11 Leutmezer F, Schernthaner C, Lurger S, Pötzelberger K, Baumgartner C. Electrocardiographic changes at the onset of epileptic seizures. Epilepsia. 2003;44(3):348-54.

12 Oliveira GR, Gondim Fde A, Hogan RE, Rola FH. Heart rate analysis differentiates dialeptic complex partial temporal lobe seizures from auras and non-epileptic seizures. Arq Neuropsiquiatr. 2007;65(3A):565-8.

13 Moseley BD, Nickels K, Britton J, Wirrell E. How common is ictal hypoxemia and bradycardia in children with partial complex and generalized convulsive seizures? Epilepsia. 2010;51(7):1219-24.

14 Evrengül H, Tanriverdi H, Dursunoglu D, Kaftan A, Kuru O, Unlu U, et al. Time and frequency domain analyses of heart rate variability in patients with epilepsy. Epilepsy Res. 2005;63(2-3):131-9.

15 Dütsch M, Hilz MJ, Devinsky O. Impaired baroreflex function in temporal lobe epilepsy. J Neurol. 2006;253(10): 1300-8.

16 El-Sayed HL, Kotby AA, Tomoum HY, El-Hadidi ES, El Behery SE, El-Ganzory AM. Non-invasive assessment of cardioregulatory autonomic functions in children with epilepsy. Acta Neurol Scand. 2007;115(6):377-84.

17 Shobha N, Satishchandra P, Sathyaprabha T, Udupa K. A study of interictal cardiac autonomic functions in patients with refractory complex partial epilepsy secondary to medial temporal lobe pathology: before and after surgery. Neurology Asia. 2007;12:69-70.

18 Oppenheimer SM, Gelb A, Girvin JP, Hachinski VC. Cardiovascular effects of human insular cortex stimulation. Neurology. 1992;42(9):1727-32.

19 Tomson T, Ericson M, Ihrman C, Lindblad LE. Heart rate variability in patients with epilepsy. Epilepsy Res. 1998;30(1):77-83.

20 Massetani R, Strata G, Galli R, Gori S, Gneri C, Limbruno U, et al. Alteration of cardiac function in patients with temporal lobe epilepsy: different roles of EEG-ECG monitoring and spectral analysis of RR variability. Epilepsia. 1997;38(3):363-9.

21 Marshall DW, Westmoreland BF, Sharbrough FW. Ictal tachycardia during temporal lobe seizures. Mayo Clin Proc. 1983;58(7):443-6.

22 Provini F, Plazzi G, Tinuper P, Vandi S, Lugaresi E, Montagna P. Nocturnal frontal lobe epilepsy. A clinical and polygraphic overview of 100 consecutive cases. Brain. 1999;122(Pt 6):1017-31.

23 Zijlmans M, Flanagan D, Gotman J. Heart rate changes and ECG abnormalities during epileptic seizures: prevalence and definition of an objective clinical sign. Epilepsia. 2002;43(8):847-54.

24 Devinsky 0. Effects of seizures on autonomic and cardiovascular function. Epilepsy Curr. 2004;4(2):43-6.

25 Nei M, Ho RT, Sperling MR. EKG abnormalities during partial seizures in refractory epilepsy. Epilepsia. 2000; 41(5):542-8.

26 Sevcencu C, Struijk JJ. Autonomic alterations and cardiac changes in epilepsy. Epilepsia. 2010;51(5):725-37.

27 Russell WJ, Morris RG, Frewin DB, Drew SE. Changes in plasma catecholamine concentrations during endotracheal intubation. Br J Anaesth. 1981;53(8):837-9. 